\title{
Acute type A aortic dissection: To crossclamp or not to crossclamp? That is the question
}

\author{
Richard J. Shemin, MD
}

See related article on pages 294-301.

The study by Lawton and colleagues ${ }^{1}$ in this issue of the Journal, "The Impact of Surgical Strategy on Survival Following Repair of Type A Aortic Dissection," retrospectively compared outcomes of operative survivors among patients treated for acute type A aortic dissection. Lawton and colleagues ${ }^{1}$ focused on the impact of surgical technique in patients operated on with a surgical strategy that included no aortic crossclamping, deep hypothermic $\left(18^{\circ} \mathrm{C}-20^{\circ} \mathrm{C}\right)$ circulatory arrest (DHCA), and the use of only antegrade restoration of body perfusion. These patients were compared with those undergoing surgical treatment with strategies that excluded 1 or more of these 3 technical steps.

Lawton and colleagues ${ }^{1}$ hypothesized that the clampless, open repair technique described in 1999 by David and coworkers ${ }^{2}$ that used hypothermic circulatory arrest was superior to other methods. David and coworkers ${ }^{1}$ theorized that femoral artery perfusion and the application of the aortic crossclamp could lead to pressurization of the false lumen, affecting the architecture of the false lumen, causing additional fenestrations and thus affecting long-term survival.

In the study of Lawton and colleagues, ${ }^{1}$ there were only 49 patients in the clampless technique group and 147 patients in the other strategies group. There were no differences in 30-day mortality or in numbers of patients with persistent distal false lumens; however, operative survivors with the clampless strategy had a statistically significant late survival advantage. The reason is obscure.

A major concern with this study is the wide variability of techniques used in the very large comparison group. There was thus a great deal of surgeon bias in this singleinstitution study from an identified aortic center. The variety of approaches attests to the lack of institutional agreement on the surgical principles tested in the study. The large variety of techniques in the control group makes the comparison and interpretation of this study difficult.

From the Ronald Reagan UCLA Medical Center; David Geffen School of Medicine, Los Angeles, Calif.

Disclosures: Author has nothing to disclose with regard to commercial support.

Received for publication April 22, 2015; accepted for publication April 25, 2015; available ahead of print June 4, 2015.

Address for reprints: Richard J. Shemin, MD, 100 UCLA Medical Plaza, Suite 730,

Los Angeles, CA 90095 (E-mail: Rshemin@mednet.ucla.edu).

J Thorac Cardiovasc Surg 2015;150:302-3

$0022-5223 / \$ 36.00$

Copyright (c) 2015 by The American Association for Thoracic Surgery

http://dx.doi.org/10.1016/j.jtcvs.2015.04.038
In addition, there were only 49 patients in the group in which all 3 key operative techniques that Lawton and colleagues ${ }^{1}$ hypothesized would result in improved outcomes were used. Ideally, a propensity-matching statistic technique should be used to perform such a comparison. Lawton and colleagues $^{1}$ chose not to propensity match the groups because of the concern that they would have only 49 pairs. The multivariable analysis performed thus leads to uncertainty.

There are more questions to consider from this study than answers derived from the data about the clamp strategy.

The surgical treatment of acute type A aortic dissection has evolved. The published literature is confusing, and most studies are not comparable. The clinical presentation and critical nature of the condition are varied and precipitate an emergency operation.

Lawton and colleagues ${ }^{1}$ emphasize the article by David and coworkers ${ }^{2}$ in 1999. This article speculated about the impact of femoral artery cardiopulmonary bypass (CPB) perfusion and the early application of an aortic crossclamp, possibly leading to false-lumen pressurization, would cause further injury to the aorta, and increased distal fenestrations. The improvement in operative and late mortality in the article of David and coworkers ${ }^{2}$ was not statistically significant. The principles of reestablishing antegrade CPB perfusion and inspection of the aortic arch during DHCA are the important surgical maneuvers. Axillary artery cannulation and antegrade cerebral perfusion were not performed in the study of David and coworkers ${ }^{2}$ or in that of Lawton and colleagues. ${ }^{1}$

Leaving patients with residual dissection is a serious issue with long-term risks and consequences for future aneurysm of the false lumen. It is a frequent occurrence despite resection of all tears and reconstitution of all aortic layers. The distal reentry sites can become entry sites, maintaining distal false-lumen pressurization and flow.

A major clinical issue resulting from not applying the crossclamp until achievement of DHCA temperature is aortic insufficiency and left ventricular distention, which often occurs when the heart fibrillates during cooling but before achievement of the appropriate DHCA temperature. Left ventricular and pulmonary arterial venting is often not adequate to achieve left ventricular decompression. The application of the aortic crossclamp with cardioplegic arrest 
becomes necessary. In addition, delay of crossclamping until DHCA temperature has been reached increases the length of the cardiopulmonary bypass period.

I believe that retrograde femoral artery perfusion should be avoided in patients with acute type A aortic dissection. Antegrade perfusion through a graft anastomosed to the right axillary artery rarely produces malperfusion or overpressurization of the false lumen with the ascending aorta crossclamped. With the clamp applied during cooling to the goal temperature for circulatory arrest, the proximal part of the repair, including resection of the ascending aorta and either aortic root repair or root and valve replacement, can be begun.

Once the goal temperature has been achieved for circulatory arrest, the proximal part of the procedure is stopped. The electroencephalogram has been isoelectric for more than 30 minutes at this time. The head is lowered, a clamp is placed on the innominate artery, and $18^{\circ} \mathrm{C}$ antegrade cerebral perfusion is begun with cerebral oxygen saturation monitoring.

The aortic crossclamp is removed, and the clamp site and aortic arch are inspected for tears. During antegrade cerebral perfusion, if the cerebral saturation drops on the left side, implying an incomplete circle of Willis, then direct cannulation and perfusion with a balloon catheter of the left carotid artery is commenced to restore equal bilateral saturations.

Any tears in the arch need to be resected. A hemiarch or complete arch replacement is performed. The layers in the aorta need to be reconstituted with biologic glue and felt before graft anastomosis. Flow into the arch vessels can be accomplished with the hemiarch technique, with individual grafts to the arch vessels if a multiple branch graft is used, or with a Carrel patch of the arch vessels after reconstituting all layers of the aorta, with a distal end-to-end anastomosis.

Then axillary artery CBP perfusion allows systemic antegrade flow to be reestablished. If adequate flows are not achieved during rewarming, a previously established $\mathrm{Y}$ from the arterial circuit allows the arch graft to be cannulated to enhance antegrade perfusion flow rates.
With systematic flow and rewarming started, the proximal portion of the operation is completed. The operation is completed with anastomosing the arch and the aortic root graft.

Thus, the major surgical maneuver to minimize malperfusion and false-lumen trauma is to avoid retrograde perfusion with femoral cannulation. Right axillary cannulation through a graft allows antegrade perfusion and an easy approach to cerebral protection with antegrade cerebral perfusion.

The application of the crossclamp with axillary antegrade perfusion is not a major issue. Careful transesophageal echocardiographic observation of flow in the true and false lumens as well as of intimal flap movement should be observed. The time saved by clamping during the cooling period allows an earlier start on the proximal portion of the operation, thus saving overall cardiopulmonary bypass time. During circulatory arrest, the clamp site is routinely resected and all aortic arch tears are resected.

The ideal study to prove the best surgical approach to CPB perfusion for cerebral protection, prevention of malperfusion of the distal aorta, and aortic trauma is unlikely to be performed. Surgical principles and best practices have emerged, so that this lethal and devastating disease can be repaired with improved early and late results.

To clamp or not to clamp remains the question. If one does use femoral cannulation, then not applying the crossclamp until achieving circulatory arrest is prudent. With axillary cannulation, one achieves antegrade perfusion so that early crossclamping can be safely performed, with the advantage of saving operative time. The clamp site must be inspected during circulatory arrest. Antegrade cerebral perfusion has proved to be an excellent technique and is facilitated by right axillary cannulation. Most importantly, establishment of antegrade $\mathrm{CPB}$ perfusion after circulatory arrest is mandatory in all cases to minimize distal aorta trauma.

\section{References}

1. Lawton JS, Liu J, Kulshrestha K, Moon MR, Damiano RJ Jr, Maniar H, Pasque MK. The impact of surgical strategy on survival after repair of type A aortic dissection. J Thorac Cardiovasc Surg. 2015;150:294-301.

2. David TE, Armstrong S, Ivanov J, Barnard S. Surgery for acute type A aortic dissection. Ann Thorac Surg. 1999;67:1999-2001; discussion 2014-9. 